\title{
Integrated Pressure-Fed Liquid Oxygen / Methane Propulsion Systems - Morpheus Experience, MARE, and Future Applications
}

\author{
Eric Hurlbert, Robert Morehead, John C. Melcher, Matt Atwell \\ NASA Johnson Space Center
}

\begin{abstract}
An integrated liquid oxygen (LOx) and methane propulsion system where common propellants are fed to the reaction control system and main engines offers advantages in performance, simplicity, reliability, and reusability. LOx/Methane provides new capabilities to use propellants that are manufactured on the Mars surface for ascent return and to integrate with power and life support systems. The clean burning, non-toxic, high vapor pressure propellants provide significant advantages for reliable ignition in a space vacuum, and for reliable safing or purging of a space-based vehicle. The NASA Advanced Exploration Systems (AES) Morpheus lander demonstrated many of these key attributes as it completed over 65 tests including 15 flights through 2014. Morpheus is a prototype of LOx/Methane propellant lander vehicle with a fully integrated propulsion system. The Morpheus lander flight demonstrations led to the proposal to use LOx/Methane for a Discovery class mission, named Moon Aging Regolith Experiment (MARE) to land an in-situ science payload for Southwest Research Institute on the Lunar surface. Lox/Methane is extensible to human spacecraft for many transportation elements of a Mars architecture. This paper discusses LOx/Methane propulsion systems in regards to trade studies, the Morpheus project experience, the MARE NAVIS (NASA Autonomous Vehicle for In-situ Science) lander, and future possible applications. The paper also discusses technology research and development needs for Lox/Methane propulsion systems.
\end{abstract}

\section{Extended Abstract}

An integrated liquid oxygen (LOx) and methane propulsion system where common propellants are fed to the reaction control system and main engines offers advantages in performance, simplicity, reliability, and reusability. LOx/Methane provides new capabilities to use propellants that are manufactured on the Mars surface for ascent return and to integrate with power and life support systems. The clean burning, non-toxic, high vapor pressure propellants provide significant advantages for reliable ignition in a space vacuum and for reliable purging of a spacebased vehicle. The roots of Lox/LCH4 propulsion system technology have a long history. Since at least the 1970's, as a replacement for toxic propellants and to achieve high performance spacecraft, efforts have been made to develop technologies needed to store and use cryogenic propellants in the space,. There has been numerous technologies programs related to suitable non-toxic propellants for use in thrusters needed for spacecraft, including even liquid hydrogen 
and liquid oxygen. However liquid hydrogen for spacecraft propulsion was determined to be very complex due to difficulties in long duration storage and the need for engine pumps. The hydrogen boil-off mass, large $\mathrm{H} 2$ tank volumes, and complexity of pumps that required redundancy resulted in a high spacecraft dry mass and cost which offset the high specific impulse. In 1980's, liquid methane was identified in a series of studies and tests, as a good fuel given its clean burning, non-sooting, space storability, capability to be pressure-fed, and commonality with LO2. $[1,2,3,4,5]$ In 1990's, the shuttle upgrade and NGLT programs conducted technology and advanced development for Lox based propulsion systems using both ethanol and methane for fuels. [6] In 2006-2010, NASA invested over \$40M into Lox/LCH4 propulsion and cryogenic fluid management systems. The NASA projects were called Cryogenic Fluid Management (CFM) and Propulsion and Cryogenics Advanced Development (PCAD). [7] .

Natural Gas) is another form of "Green Propellant" that replaces toxic propellants for certain applications where bipropellants are typically used. [8] It is complimentary to propellants used in the GPIM. It is a green propellant that is well suited for deep space and planetary exploration landers with higher cargo capacity, high thrust, and high delta-V requirements. LOX/LCH4 has a higher thrust capability than hydrazine and electric propulsion systems. It is not intended to be a satellite propellant replacement. LOX/ LCH4 reduces spacecraft total mass due to higher Isp when combined with composite propellant tanks and GHe stored cold at propellant temperatures. LOX/LCH4 is a space storable propellant combination. No heaters are required as with earth storable propellants and no active cooling as with LH2. In certain environments, LOX and methane can be stored indefinitely at temperatures of $90-120 \mathrm{~K}$ in equilibrium with deep space environments and for months in other orbits. Lox is compatible with many materials and methane is compatible with nearly all materials. Both are non-corrosive. They are clean burning, non-sooting, high vapor pressure propellants that do not coat sensitive optics or contaminate surfaces as does MMH/NTO. LOX/LCH4 propellants are capable of being pressure-fed which provides inherent reliability advantages. The propellants are fully non-toxic and low cost which enables rapid loading, testing, and turnaround operations of the spacecraft and subsystems. Automated spacecraft loading can be done in parallel with the launch vehicle, similar to LOX/LH2 upper stages, but without the issues of LH2 or the issues of toxic propellants. No preloading of hazardous propellants onto the spacecraft is required at an offsite facility. The inert spacecraft is transported and integrated in the launch vehicle. For loading at the launch pad, mobile propellant storage tanks, propellant transfer lines on servicing tower, and cryogenic fluid couplings will be required.

From 2011-2014, the NASA Advanced Exploration Systems (AES) Morpheus lander demonstrated many of these key attributes as it completed over 65 tests including 15 flights through 2014. Morpheus is a prototype of LOx/Methane propellant lander vehicle with a fully integrated propulsion system. The Morpheus propulsion system and vehicle was designed to provide 120 seconds of flight while carrying a $400 \mathrm{lbm}$ payload. The payload consisted of Autonomous Landing and Hazard Avoidance Technology (ALHAT) which mapped the terrain in 3 dimensions, autonomously selected the safe landing site, and then navigated to safe site. The propulsion system consisted of a gimbaled $5400 \mathrm{lbf}$ thrust engine with 4:1 throttling and four $\sim 20 \mathrm{lbf}$ max roll control thrusters. This was fed from 4 aluminum propellant tanks that were 
operated in blowdown. After the free flights were completed, the vehicle was modified to include a $2800 \mathrm{lbf}$ (vac) engine and a cold GHe pressurization system.
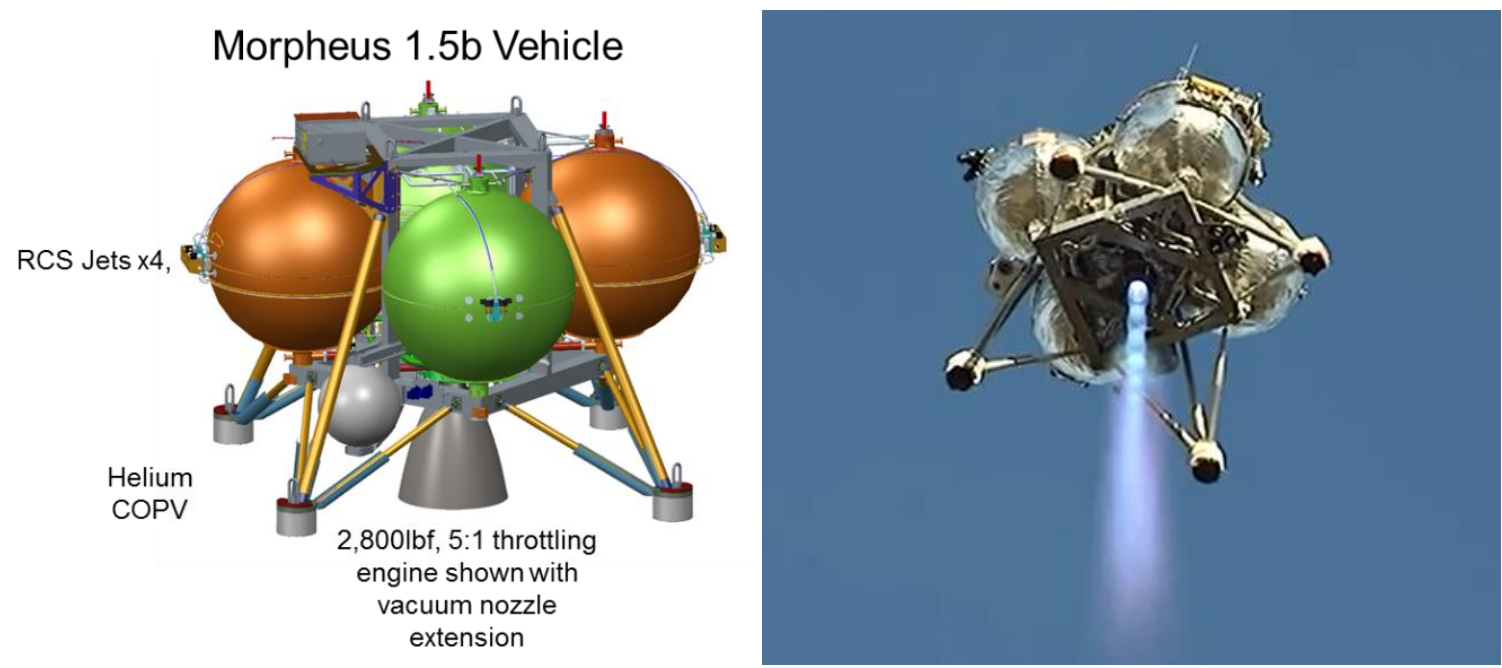

The Morpheus lander flight demonstrations led to the proposal to use LOx/Methane for a Discovery class mission, named Moon Aging Regolith Experiment (MARE) to land an in-situ science payload for Southwest Research Institute on the Lunar surface. The MARE proposal consisted on the NAVIS (NASA Autonomous Vehicle for In-situ Science) lander and the science experiment MARE. Several modifications to the Morpheus vehicle are required. This vehicle uses the $2800 \mathrm{lbf}$ thrust engine and $5 \mathrm{lbf}$ RCS thrusters. The tanks were reduced slightly in diameter to 45 inches. The seals and fittings were upgraded to flight type. The insulation was replaced with multi-layer insulation designed for space vacuum.

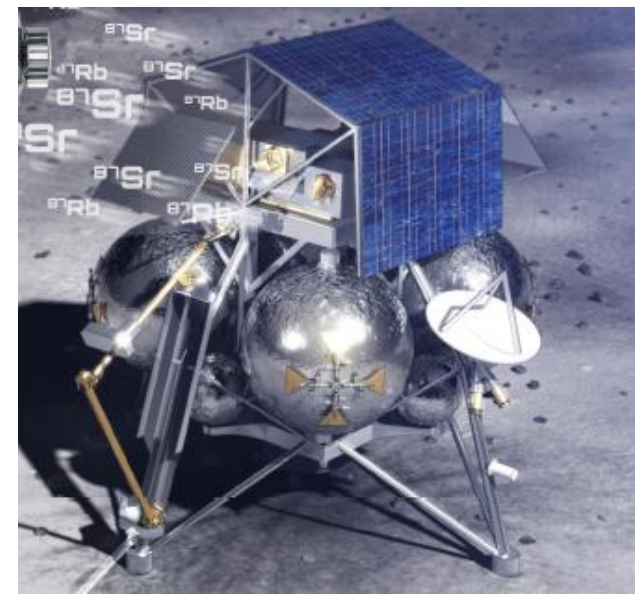

In 2015, a different NASA study examined the potential for a pressure-fed LOx/Methane system to use for Mars ascent vehicle was completed. The system uses a similar architecture to 
Morpheus and MARE, but scales up the thrust to 50,000 lbf and adds mechanical redundancy similar to the Apollo ascent stage. A mass fraction of propellant to propulsion system and primary structure dry mass total of 0.85 is required to be competitive with pump-fed solutions. The advantage of Mars ascent is that the atmospheric pressure is low (0.1 psia) and gravity is $3 / 8$ that of earth. These both mean that a pressure-fed solution is more feasible, as compared with earth launch vehicle. Future trades with pump-fed system solutions is required. The use in-situ produced and stored propellants requires very low heat leaks in the Martian atmosphere.

Lightweight vacuum jacketing technologies is required. For this design study, a tension strap system similar to Space Shuttle fuel cell tanks was used.
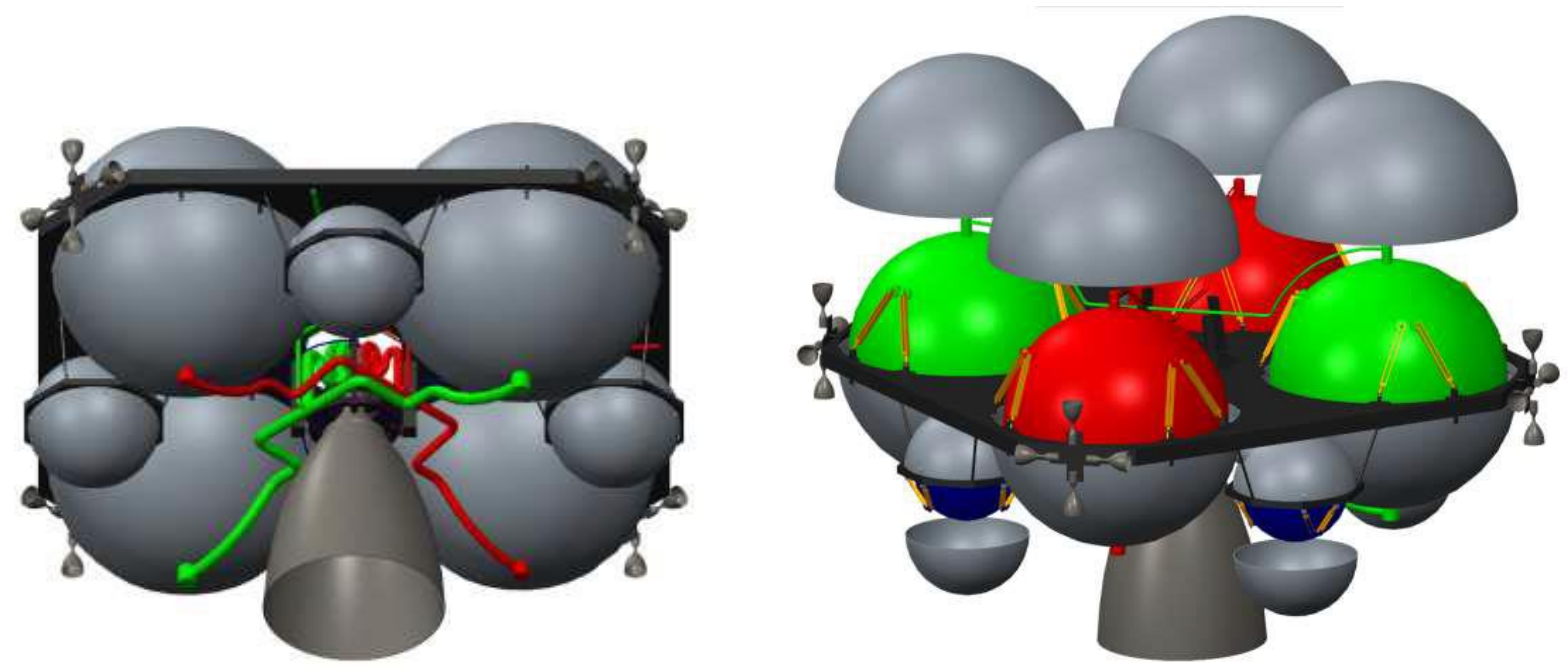

The technology research and development needs for Lox/Methane propulsion systems include vacuum jacketing, composite propellant tanks, regeneratively-cooled throttling engines, and cryogenic reaction control systems. These technologies will support many possible applications for $\mathrm{LOx} / \mathrm{LCH} 4$ for spacecraft. 